\title{
Schlaganfallprophylaxe bei Vorhofflimmern
}

\section{Studie bescheinigt Rivaroxaban günstiges Sicherheitsprofil}

\begin{abstract}
_ Schlaganfälle verlaufen laut Prof. Martin Grond, Siegen, besonders schwer, wenn sie durch Vorhofflimmern (VHF) bedingt sind. Dies unterstreiche die Bedeutung der prophylaktischen Antikoagulation, wofür zunehmend die neuen oralen Antikoagulanzien (NOAK) zum Einsatz kämen.

Sie hätten in Studien ein geringeres Risiko für schwerwiegende Blutungen gezeigt als Vitamin-K-Antagonisten (VKA). Das gelte insbesondere für hämorrhagische Schlaganfälle, die unter NOAK signifikant $(\mathrm{p}<0,0001)$ seltener vorkommen als unter VKA [Ruff CT et al. Lancet. 2014;383:955-62]. Der Faktor-Xa-Hemmer Rivaroxaban (Xarelto ${ }^{\circ}$ )
\end{abstract}

habe sein positives Nutzen-Risiko-Profil und das niedrigere Risiko für schwere Blutungen bereits in der Zulassungsstudie ROCKET AF belegt.

\section{Daten aus dem klinischen Alltag}

Nun untermauert die Studie XANTUS [Camm AJ et al., Eur Heart J 2015, online 1 September 2015. doi:10.1093/eurheartj/ ehv466] das günstige Ergebnis hinsichtlich der Blutungskomplikationen auch im klinischen Alltag, berichtete Prof. Matthias Leschke, Esslingen. Mehr als 6.700 Patienten mit nicht-valvulärem Vorhofflimmern wurden für die Studie mit Rivaroxaban behandelt. Es ergab sich eine Inzidenz schwerer Blutungs- ereignisse von lediglich 2,1\% pro Patientenjahr. Besonders bemerkenswert war laut Leschke die Tatsache, dass bei $96,1 \%$ der Patienten weder eine medikationsbedingte schwere Blutung noch ein symptomatisches thromboembolisches Ereignis noch ein Todesfall jedweder Ursache auftrat.

„Die aktuellen Daten bekräftigen somit das positive Sicherheitsprofil von Rivaroxaban, das sich im klinischen Alltag sogar noch günstiger darstellt als in der Zulassungsstudie ROCKET AF“, resümierte der Kardiologe.

Christine Vetter

- Presseveranstaltung „ImPRESSionen - Antikoagulation live"; Essen, 29. Februar 2016 (Veranstalter: Bayer Vital)

\section{Rückenschmerzen mit neuropathischer Komponente}

\section{Retardiertes Oxycodon/Naloxon in Studie Tapentadol überlegen}

\begin{abstract}
- Die Fixkombination aus retardiertem Oxycodon und retardiertem Naloxon hat sich in einer aktuellen nicht-interventionellen Studie an Patienten mit lange bestehenden, starken Rückenschmerzen mit neuropathischem Anteil gegenüber Tapentadol als überlegen erwiesen. Die prospektive, randomisierte, offene, multizentrische OXYNTA-Studie mit verblindetem Endpunkt wurde von der Deutschen Gesellschaft für Schmerzmedizin durchgeführt, wie PD Dr. Michael Überall, Nürnberg, berichtete.
\end{abstract}

\section{Kombinierter Studienendpunkt}

Als Wirksamkeitsendpunkte gingen klinisch relevante Verbesserungen ( $\geq 30 \%$ ) der Rückenschmerzen laut Back Pain Intensity Index, der schmerzbedingten Funktionseinschränkungen im Alltag laut modifiziertem Pain Disability Index sowie der Lebensqualität laut EQ-5D-3L-
Fragebogen in die Bewertung ein. Nebenwirkungsendpunkte waren die Therapietreue bis zum Studienende, eine normale Darmtätigkeit laut Bowel Function Index und das Fehlen von ZNS-Nebenwirkungen.

$\mathrm{Zu}$ Beginn wiesen die bei allen Patienten chronifizierten Schmerzen im 24-Stunden-Mittel einen Schmerzindex von $55 \mathrm{~mm}$ auf der visuellen Analogskala auf. Beide Therapieregime zeigten ein vergleichbares Sicherheits- und Verträglichkeitsprofil. Den kombinierten Endpunkt erreichten nach zwölf Wochen $39,8 \%$ der Patienten mit retardiertem Oxycodon/Naloxon (Targin ${ }^{\circ}$ ) und 25,6\% unter Tapentadol $(\mathrm{p}=0,014)$.

Die Studienergebnisse decken sich laut dem Schmerzmediziner Dr. Jakob Emrich, Ludwigshafen, mit den Erfahrungen in der Praxis.

Dr. Wiebke Kathmann

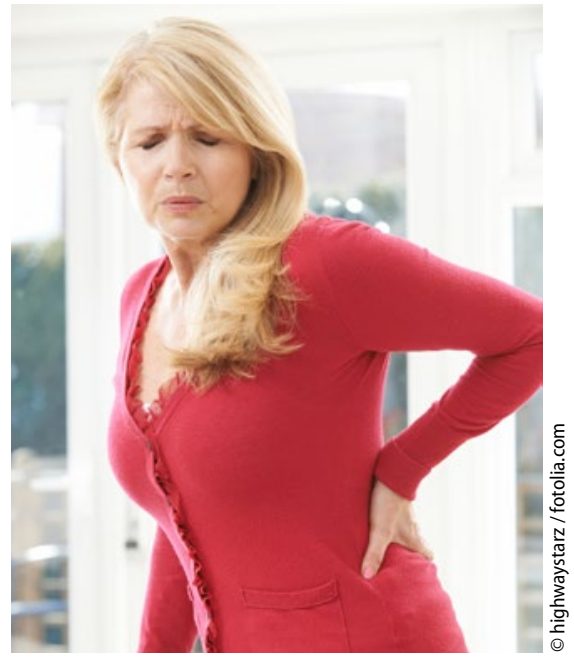

Chronische Rückenschmerzen mindert die Lebensqualität der Patienten.

- Pressegespräch „Neue OXYNTA-Daten zu Targin ${ }^{\star}:$ Schmerzvielfalt effektiv begegnen" beim 27. Deutschen interdisziplinären Schmerz-und Palliativkongress; Frankfurt am Main, März 2016 (Veranstalter:Mundipharma) 